\title{
PRIMEIROS REGISTROS DOS GOLFINHUS STENELLA COERULEOALBA, STENELLA CFR. PLAGIODON E STENO BREDANENSIS PARA O SUL DO BRASIL, COM NOTAS OSTEOLOGICAS*
}

MARIA CRISTINA PINEDO e HUGO P. CASTELLO

Base Oceanográfica A tlântica, Fundação Universidade do Rio Grande, Rio Grande, RS, Brasil

\section{SYNOPSIS}

scenella coeruleoalba is a dolphin widely distributed in temperate and tropical waters. This species is recorded for our marine fauna on the basis of a $2.35 \mathrm{~m}$ long specimen found at $20 \mathrm{~km}$ North of the East rocky jetty on the access channel into Lagoa dos Patos, RS, (MORG 064) and also from a sightning in front of Santa Catarina coast. Its vertebral formula is: C $7+D 14+L 21+$ Ca $34=76$, with 23 Chevron bones. The number of alveoli is $48-48 / 43-42$, its presents 14 pairs of ribs, 8 of them double-headed. The skull measurements of this specimen are compared with other three from the Southern Hemisphere. Through colour slides obtained in front of Rio de Janeiro coasts, Steno bradanensis was recorded. This dolphin also presents a wide distribution similar to the previous species. During the first cruise of the R/V "Atlantico Sul" two pods of the spotted dolphin. Stenella $c f r$. plagiodon were sighted and photographed. This species can be easily identified on the basis of the clear brown tegument with numerous white rounded spots, in adults. One of the skull (MCNRS 026) studied may belong to this species.

\section{Introdução}

Registra-se a ocorrência de 3 espécies de golf inhos (Delphinidae) para o Atlântico Sul Ocidental brasileiro, Stenella coeruleoalba, Stenella cfr. plagiodon e Steno bredanensis, até agora não citadas na lista de cetáceos elaborada por Carvalho (1975).

Stenella coeruleoalba (Meyen, 1833)

Material estudado: 1 ex.. $2.35 \mathrm{~m}$ comp. tot., encalhado $20 \mathrm{~km} \mathrm{~N}$. Molhe Leste do Canal de Acesso à Lagoa dos Patos, RS (MORG 064), 22/11/77. Col. H.P.C. e M.C.P.

1 slide colorido, manada de golfinhos brincando na proa do "Atlántico Sul", $10 / 12$ milhas da costa de Tramandaí $\left(30^{\circ} 00^{\prime} \mathrm{S}\right.$ $49^{\circ} 52^{\prime}$ 'W, prof. $\left.36 \mathrm{~m}\right), 23 / 02 / 78$, obtido pelo Prof. R. Perez Habiaga, BOA-FURG.

Distribuição geográfica: O tipo desta espécie foi descrito em base a um espécimen fêma de $1.67 \mathrm{~m}$, arpoado frente ao Rio de $\mathrm{La}$ Plata (Meyen. 1833) Encontra-se distribuido em águas temperadas subtropicais e tropicais ao redor do mundo (Rice, 1977). No Atlântico Sul Ocidental os unicos registros sâo para: águas do Balneário Solymar, Uruguay $\left(34^{\circ} 49^{\prime} \mathrm{S}-55^{\circ} 55^{\top} \mathrm{W}\right), 08 / 59$, (MNHN Montevideo $1305)$ e para Miramar, Pcia. de Buenos Aires, Argentina ( $\left.38^{\circ} 17^{\prime} S\right)$ (MACN 2625) ambos já mencionados por Brownell \& Praderi (1976).

Coloração: Em estado fresco o dorso é cinza escuro ou azulado, sendo as regiōes laterais e o ventre cinza. Apresenta distintas listras negras desde: 1 ) olho ao anus; 2 ) olho até a nadadeira peitoral. Marca negra distinta desde a região anterior da nadadeira dorsal até a origem da peitoral (Leatherwood et al., 1976). No slide obtido pelo Prof. Habiaga nota-se com nitidez uma marca clara que se origina na região ventral e termina embaixo da dorsal em forma de ponta de lança, que em inglés é conhecida como "brush mark", "flank pigment" ou "spinal blaze", de acordo com Morze Bruyns (1974).

Morfologia externa: Pode alcançar $2,70 \mathrm{~m}$ ou algo mais de comprimento, sendo seu corpo alongado e com nadadeira dorsal levemente falcada. O adiantado estado de decomposição de nosso exemplar impediu a visualização dos padrões de coloração característico da espécie.

Medidas corporais: Comprimento total: $2,35 \mathrm{~m}$; dist. orig. tocinho a comissura bucal: $0,33 \mathrm{~m}$; dist. orig. focinho ao olho: $0.38 \mathrm{~m}$; dist. orig. focinho ao respiradouro: $0,36 \mathrm{~m}$; dist. orig. focinho a orig. peitoral: $0,55 \mathrm{~m}$; dist. orig. focinho a orig. dorsal: $1,05 \mathrm{~m}$ comprimento anterior da peitoral: $0,29 \mathrm{~m}$; comp. post. peitoral: $0,21 \mathrm{~m}$; alt. nad. dorsal: $0,16 \mathrm{~m}$; larg. da cauda: $0,35 \mathrm{~m}$.

Medidas do crânio: Foram tomadas as medidas do crânio e mandibula (Tab. I) e comparadas com as obtidas por Brownell \& Praderi (opus cit.) para o exemplar da Argentina (MACN 2625) e com as do exemplar de Nova Zelândia (Dominion Mus. 30) dadas por Baker \& Stephensosn (1982) (Fig. 4).

Nota-se uma protuberância óssea supra-occipital acentuada, localizada entre o nasal e o occipital. A fossa temporal apresenta-se de forma ovalada, sendo seu comprimento maior que a altura (66x56mm respectivamente).

O número de dentes varia de $42-48$ pares em ambas mandí. bulas, sendo o número dado como comum para esta espécie de 39 a 47 pares, e de $48-50$ pares no exemplar tipo.

Esqueleto: A fórmula vertebral é: C7 + D14 + L21 + Ca34 $=$ $=76 . \mathrm{O}$ atlas $\mathrm{e} \mathrm{o}$ axis estão fusionados. $\mathrm{A}$ fórmula vertebral dada para S. coeruleoalba por Nishiwaki (1972) é C7 + D15 + L2 +

- Contribuição da Base Oceanográfica Atlântica-no 8.
+ Ca $35=79$. O número de ossos de chevron é de $23 ;$ o primeiro deles encontra-se articulado entre as vertebras $42 \mathrm{a}$. e $43 \mathrm{a}$. ou seja, a vértebra 42 a. é a primeira caudal. Apresenta 14 nares de costelas. das quais 8 são de dupla cabeça e 6 simples (Tab. II). A morfologia dos ossos hióides, esterno e escápula estão representados nas Figs. 1,2 e 3 e suas respectivas medidas na Tabela II.

$\mathrm{O}$ esterno encontra-se dividido em duas peças.

Observação: A foto do crânio do exemplar MORG 064 foi observada pelo Dr. W. F. Ferrin do S. W. Fish. Center, La Jolla, California, USA, que confirmou a identificação do material. O exemplar citado trata-se do primeiro a ser coletado completo e estudado, perto da localidade típica da espécie, ou seja, o Rio de La Plata.

\section{Stenella cfr. plagiodon}

Material estudado: 1 crânio sem mandíbula, Torres, RS, 02/1956, Rio Grande do Sul (MCNRS 026). Col. Zwenegon. 3 fotos coloridas, manada de golfinhos nadando na proa do "Atlântico Sul" $10 / 12$ milhas da costa de Tramandaí $\left(30^{\circ} 00^{\prime} \mathrm{S}-49^{\circ} 52^{\prime} \mathrm{W}\right.$ ', prof. $36 \mathrm{~m}), 23 / 02 / 78$ obtidas pelo Prof. N. Gianuca - BOA-FJ'RG.

Distribuição geográfica: É uma espécie comum, encontrada em águas tropicais e temperadas do Atlântico Norte Ocidental, desde New Jersey até o Panamá, incluindo o Golfo do México (Caldwell \& Caldwell, 1966).

Até o presente momento $\mathrm{n}_{\mathrm{u}}$ ) existe registros da espécie para o Atlântico Sul Ocidental, a não ser um crânio examinado por Brownell \& Praderi (opus cit.) procedente de Mar de Plata, Pcia. de Buenos Aires, Argentina ( $38^{\circ} 00^{\prime} \mathrm{S}$ ) (MACN 23-46). Contudo os autores, tendo em vista o pouco conhecimento sistemático das espécies, se referem a esse crânio como pertencente ao complexo dos golfinhos estriados ( $S$. attenuata, $S$. dubia, $S$. frontalis e $S$. plagiodon).

Coloração: $S$. plagiodon apresenta aorsalmente numerosas pintas brancas e ventralmente pintas escuras, sendo que esta coloração incrementa-se com a idade. Os juvenis são de coloração uniforme (Leatherwood et all., 1976).

No caso de $S$, attenuata, espécie do Pacífico, os machos só atingem a completa pigmentação aos 11 anos de idade e a fêmea aos 9 anos (Kasuya, Miyasaki \& Dawbin, 1974).

Observação: O crânio examinado (MCNRS 026), embora semelhante a $S$. coeruleoalba, apresenta características distintas, tais como: a forma da fossa temporal é triangular, sendo seu comprimento maior que a altura, em contraste com a de coeruleoalba que se apresenta ovalada. As larguras do pré-orbital e orbital são bem menores, o mesmo acontecendo para a largura do arco zigomástico (Tab. I). A foto deste crânio e as medidas foram enviadas ao Dr. Perrin, que estima que o mesmo pertenceria à espécie $S$. cfr. plagiodon. Examinando uma das fotos coloridas nota-se a presença de uma manada com 10 golfinhos, sendo que dois deles apresentam as pintas características, e os outros uma coloração uniforme. Estimamos que o processo de pigmentação deve ser semelhante ao que ocorre com a espécie do Pacífico, $S$. attenuata.

\section{Steno bredanensis}

Material estudado: 1 slide colorido, 5 exemplares de golfinho nadando na proa do N/Pq. "Riobaldo" da SUDEPE entre Cabo Frio e Baia da Guanabara (RJ), no primeiro semestre de 1974, obtido pelo Prof. Mitsuo Yesaki.

Distribuição geográfica: Registros de Steno bredanensis (rougth toothed dolphin) para o Atlântico N.O. e áreas adjacentes 



Fig. 4. Medidas do crânio de $S$. couruleoalba e $S$. cfr. plagiodon.

$\mathrm{A}=$ MORG. $064 ; \mathrm{B}=\mathrm{D} . \mathrm{M} .30 ; \mathrm{C}=\mathrm{MACN} .2625 ; \mathrm{D}=$ MCNRS. 026

Obs.: As medidas estão em ordem descrescente; tomou-se como referência o exemplar MORG. 064.

As abcissas do gráficos apresentam numeração de acordo com a tabela I. 
Tab. I - Quadro comparativo de medidas do crânio (milímetros) e número de dentes de $S$. coeruleoalba e $S$. cfr. plagiodon

\section{ME DIDAS}

01. Comprimento côndilo-basal

02. Comprimento da mandíbula

03. Ponta do rostro às narinas

04. Comprimento do rostro

05. Comprimento da linha dentária superior direita

06. Comprimento da linha dentária superior esquerda

07. Largura pós-orbital

08. Comprimento da linha dentária inferior direita

09. Comprimento da linha dentária inferior esquerda

10. Largura do arco zigomático

11. Largura do pré-orbital

12. Largura do orbital

13. Largura da caixa craniana entre os parietais

14. Largura rostro na base

15. Larg. máxima do pré-maxilar

16. Larg. rostro a $60 \mathrm{~mm}$ da base

17. Altura do coronóide

18 Comp. da fossa temporal

19. Larg. do rostro no meio

20. Comp. da sínfisis mandibular

21. Altura da fossa temporal

22. Com. do rostro a $3 / 4$ de distância da base

23. Larg. do pré-maxilar ao meio

24. Número de alvéolos sup. direita

25. Número de alvéolos sup. esquerda

26. Número de alvéolos inf. direita

27. Número de alvéolos inf. esquerda
MORG.

064

D.M.

30

MACNBA

2625

MCNRS

$\mathrm{mm} \quad \% \mathrm{CBL} \mathrm{mm} \quad \% \mathrm{CBL} \mathrm{mm} \% \mathrm{CBL} \mathrm{mm} \% \mathrm{CBL}$

$\begin{array}{cccccccc}479 & 100 & 479 & 100 & 442 & 100 & 440 & 100 \\ 406 & 84.7 & 416 & 86.8 & 374 & 84.6 & - & - \\ 348 & 72.6 & 341 & 71.1 & 305 & 69.0 & 311 & 70.6 \\ 293 & 61.1 & 280 & 58.4 & 260 & 58.8 & 270 & 61.3 \\ & & & & & & & \\ 245 & 51.1 & 230 & 48.0 & 230 & 52.0 & 222 & 50.4 \\ 242 & 50.5 & 230 & 48.0 & 229 & 51.8 & 224 & 50.9 \\ 228 & 47.5 & 231 & 48.2 & 206 & 46.6 & 186 & 42.2\end{array}$

226

221

214

210

208

193

117

47.1

237

49.4

226

51.1

61

60

46.1

239

44.6

227

43.8

178

49.8

226

47.3

209

51.1

43.4

37.1

185

47.2

41.8

185

56

40.

24.4

200

$-$

191

43.2

119

$17.7 \quad 88$

41.7

150

33.9

25.0

111

25.1

18.3

$16.0 \quad 84$

17.5

-

$-$

$14.6 \quad 76.5$

15.9

70

15.8

$13.7 \quad 61$

12.7

67

15.1

16.0

13.9

$12.7 \quad 67$

13.9
12.3

12.5

59

12.9

71

13.3

59

11.3

11.6

62

10.4

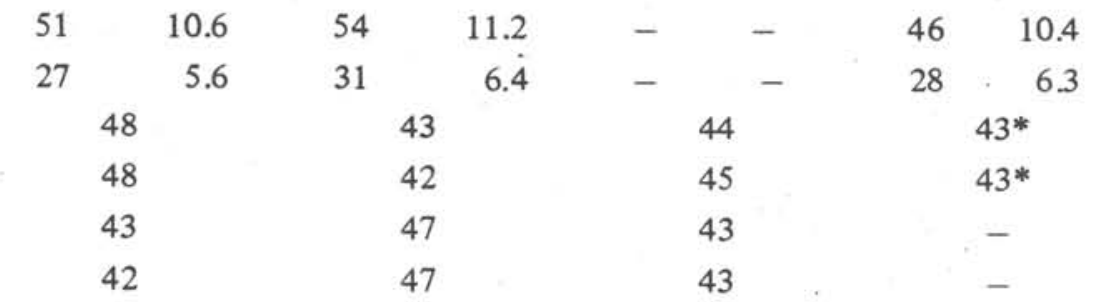

* Mais $3 \mathrm{~cm}$ do rostro sem dentes 
Tab. II - Medidas do osso hióide, esterno, escapulares e costelas de S. coeruleoalba (MORG 064), em mm.

\section{Osso hióide}

01. Comprimento reto

02. Maior altura

03. Altura no centro

04. Profundidade da fossa

05. Altura no meio da asa direita

06. Altura no meio da asa esquerda

07. Espessura no meio da asa direita

08. Espessura no meio da asa esquerda

09. Comprimento da asa direita

10. Comprimento da asa esquerda 19

11. Comprimento total do stylohyal 90

12. Altura no meio do stylohyal 16

13. Espessura no meio

Esterno

01. Comprimento

Peça

maior

02. Largura máxima

03. Largura na parte anterior

04. Comprimento da cavidade

\section{Escapulares}

01. Largura máxima

02. Altura máxima

03. Comprimento do coracóide

04. Largura máxima do coracóide

05. Comprimento da fossa glenóide

06. Largura da fossa glenóide

07. Comprimento do acrômio

08. Largura máxima do acrômio
Direito

220

145

45

Quebrado

28

22

53

52

\section{Costelas}

Número

2

5

8

11

14

$\begin{array}{cc}\text { Direita } & \text { Esquerda } \\ 202 & 203 \\ 325 & 325 \\ 320 & 331 \\ 298 & 293 \\ 242 & \end{array}$

Esquerdo

30

28

22

50

50 
inclui espécimens desde o Caribe, ate o Golfo do México (Layne, 1965: Richardson, 1973). Há registros também para o Pacífico Norte Ocidental, fazendo parte a espécie de sua fauna tropical (Leatherwood \& Evans, 1972). São também conhecidos registros de exemplares encalhados na Costa Ocidental da África, ilhas de Cabo Verde, de Steno sp., provavelmente $S$. bredanendis (Cadenat, 1948).

Morfologia externa e coloração: Pode alcançar $2,40 \mathrm{~m}$ ou algo mais em comprimento total. Suas principais características são de grandes olhos e nadadeiras peitorais, e a ausência de demarcação entre o longo bico e a cabeça, dando-the uma aparência quase cômica. Sua coloração é bastante variável: cinza no dorso com a presença ou não de pintas brancas nos lados e ventre. As nadadeiras peitorais, caudal e dorsal são escuras e o ventre é branco (Leatherwood et al., opus cit.).

\section{Bibliografia}

BAKER, A. N. \& STEPHENSON, A.B. 1972 . The ocurrence of the dolphin genus Stenella in New Zeland waters. Rec. Domin Mus. 8(1): 107-114

BROWNELL JR., R. L. \& PRADERI, R. 1976. Records of the delphinid genus Stenella in Western South Atlantic waters. Sci. Rep. Whales Res. Inst., Japan, (28) 129-135.

CADENAT, J. 1948. Notes sur les cétacés observés sur les côtes du Sénégal de 1941 a 1948. Bull. Ifan, 11:1-15.

CALDWELL, D.K. \& CALDWELL, M. C. 1966. Observations on the distribution, coloration, behavoir and audible production of the spotted dolphin Stenella plagiodon (Cope). Contr. Sci. Los Angeles Count. Mus, (104): 1-104.
CARVALHO, C.T. de 1975. Ocorrencias de mamíferos marinhos no Brasil. Bolm Téc. Inst. Flor., São Paulo, (16): 12-32.

KASUYA, T.; MIYAZAKI, N. \& DAWBIN, W. H. 1974. Growth and reproduction of Stenella attenuata in the Pacific coast of Japan. Sci. Rep. Whales. Res. Inst., Japan (26): 257-226.

LAYNE, J. N. 1965. Observation on marine mammals in Florida waters. Bull. Florida St. Mus. Biol. Sc., Gaineswille, 9(4): 131-181.

LEATHERWOOD, S. \& EVANS, W. E. 1972. The whales, dolphins and porpoises of the E.N.Pacific. A guide to their identification in the water. Nav. Und. Cent. California: TP $282 ; 175 \mathrm{p}$.

LEATHERWOOD, S.; CANDWELL, D. K. \& WINN, H. E. 1976. Whales, dolphins and porposies of the Atlantic. A guide to their identification. N.O.A.A. Tech. Rep. NMFC Circ., (396): $176 \mathrm{p}$.

MEYEN, F. J. F. 1833. Beitrage zur Zoologie, gesammlet auf einer Reise un die Erde. Nov. Act. Acad. Caes. Leop. Carol. Nat. Curios., 16(2): 551-610.

MORZER BRUYNS, W. F. J. 1974. On sigth-records of the euphrosyne dolphin, Stenella coeruleoalba (Meyen, 1843), in the Mediterranean. Mammalia, Paris 38(3): 503-507.

NISHIWAKI, M. 1972. (Marine Mammal) General Biology. In "Mammals of the sea. Biology and Medicine" (Ed. Ridway). Illinois, Ch. C. Thomas, $812 \mathrm{p}$.

RICE, D. W. 1977. A list of the marine mammals of the world. N.O.A.A. Tech. Rep. NMFS, (711): 1-15

RICHARDSON, J. I. 1973. A confirmed occurrence of the roughtoothed dolphin (Steno bredanensis) on the Atlantic coast of the United States. J. Mammal., Washington, 54(2): 275. 